\title{
ABOUT NONLINEAR CLASSIC FIELD THEORY OF CONNECTED CHARGES
}

\author{
Stanislav Podosenov ${ }^{1}$, Jaykov Foukzon ${ }^{2}$, Alexander Potapov ${ }^{3}$, and Elena \\ Men'kova ${ }^{4}$ \\ ${ }^{1}$ All-Russian Research Institute of Optophysical Measurements, Moscow, Russia \\ ${ }^{2}$ Israel Institute of Technology, Haifa, Israel \\ ${ }^{3}$ Kotel'nikov Institute of Radioengineering and Electronics of the Russian Academy of \\ Sciences, Moscow, Russia \\ ${ }^{4}$ All-Russian Research Institute of Optophysical Measurements, Moscow, Russia
}

\begin{abstract}
On the basis of an exact solution for the field of a charge in a uniformly accelerated noninertial frame of reference (NFR) and formulated "Equivalent Situation Postulate" the nonlinear electrostatic theory of bound charges has been constructed. Proposed method is outside of the flat space-time, however the curvature is not directly connected with the Einstein gravitational theory. The method proposed eliminates divergence of the proper energy and makes classical electrodynamics consistent at any sufficiently small distances.
\end{abstract}

\section{KEYWORDS}

Space-time, metric tensor, curvature tensor, frames of reference, Bell's problem, Born's rigidity, equations of structure, Minkowski, Riemann, Einstein, Lagrangian coordinates, Kristoffel

\section{INTRODUCTION}

The main difficulty of classic theory of electromagnetic field is the contradiction between assumption of charged particles stippling and their infinite own energy. Attempts of removal of this difficulty were connected with development of nonlinear theory G. Mi (1912), M Born and L. Infeld (1934) [1]. However the foundations of hese theories are based on arbitrary selection of Lagrangian and do not probe by the experiment. This is the main disadvantage of these theories. Proposed model of nonlinear theory is based on account of charge interaction creating the field with produced field. In this case the connection field keeping (in electrostatics) constant charge configuration has been taken into account. However proposed approach falls outside the plane space-time. Maxwell's equations wrote in generally covariant four-dimensional form are initial equations of the theory. They are similar as the electrodynamic equations when the gravitational field is present [2]. Instead the Einstein equations the structure equations have been used to determine the metric. These equations establish the connection between kinematic characteristics of reference system (RS) such as tensor of deformation velocities, tensor of angular speed of rotation, 4-acceleration with metric tensor RS . Likewise general theory of relativity (GTR) RS is the "totality of infinite number of bodies filling up all space similarly some "medium"' [2]. The structure equations are the compatibility equations for the existance of the 4velocity field at specified strain velocity tensors, angular velocity and first curvature vectors of the world lines of the RF basis particles.

The transition to Lagrange co-moving frame of reference (NRF) is connected in the general case with the construction of three-dimensional "physical space of the observers orthogonal to the world lines of the basis particles". In this space the medium always is at rest but the tensors characterizing the 
medium in the general case are differed from zero. When the rotations are present in the medium as it has been proved by J. Sing [3], the three-dimensional "physical space" is anholonomic.

The solution of the Pphaff equation specifying the properties of the three-dimensional comoving space of the observers is absent. These properties are considered in detail in monoographs $[4,5]$, where the theory of anholonomic transformations has been developed. This theory is closely related to the fractal theory [6]. It has been shown in [4,5] that when the rotations are present in the three-dimensilnal comoving space the partial derivatives on the space Lagrange coordinates are substituted with the directional derivatives. This results in anholonomic objects different from zero. Hence the algorithm for determination of the length of the spatial curves length locating in the "physical Lagrange space" on the basis of the fractal methods [6] is arisen. For the vortex-free motions the spatial length is determined in [7-10]. The length of the electron pulse in the linear accelerators at the Lagrange comoving RF differes on a few orders from the standard calculation which does not take into account the spatial curvature of the beam. In [8-10] the original formula for the known Bell paradox [11,12] taking into account the bending of the spatial length has been obtained. It has been proved in [13] that the transition to the hard globally uniformly accelerated NRF results in cuvature of space-time. Later these results have been repeated in [14,15] (without reference [13]). More detail the theory has been presented in monographs [4,5].

Let us formulate

\section{Equivalent Situation Postulate}

The field of a point charge, being at equilibrium in a constant electric field, is equivalent to the field from this charge in a uniformly accelerated NFR, if the constraint reaction forces, accelerating the charge and holding it immovable, are equal and opposite in direction

In classical electrodynamics the field of a point charge being at rest in an inertial frame of reference (IFR) is Coulomb's spherically symmetric one, irrespective of whether the charge is free or the sum of forces acting on it is zero. The field from a uniformly accelerated charge proves to be axially symmetric for the observer in an NFR irrespective of the method of the transition to NFR [2]. Thus, the identical physical situation, in which the charges are (identical constraint forces), results in the field with different symmetry! We try to solve this paradox in the present paper.

\section{NFR STRUCTURAL EQUATIONS}

The 4-acceleration, the strain rate tensor and the tensor of the angular velocity are the characteristics of the continuum in the four-dimensional space-time. 4-acceleration is entered into the law of motion and at the known plane metric the field of the 4-velocity and the main medium tensors are determined by the integration of the equation of motion. For the frames of reference with the specified properties one must to know the additional conditions of the main medium tensors depending on 4-velicities and 4 -accelerations. For example, the demand of the rotation absence and the Born rigidity. The number of the equations for determination of the 4-velocity becomes overdetermined and the integrability conditions must to be fulfilled. They are fulfilled when both 4 -velocities of the medium and the metric coefficients are unknown.

In the relativistic theory the universally recognized analytic definition both frames of reference and the rules determined the transition between them are absent [16]. In [13] the theory of the relativistic hard uniformly accelerated NRF realized at the Riemannian space with the constant curvature has been constructed. When NRF constructing the approach is based on the obvious demand of the absence of the deformations and stresses in the rigid body if it moves translationally in the uniform force field. The following relations have been obtained. Let us assume that a continuous medium moves in fourdimensional space-time with signature $(+---)$. The medium satisfies the expansion

$$
\nabla_{\mu} V_{v}=\Sigma_{\mu \nu}+\Omega_{\mu \nu}+V_{\mu} F_{v^{\prime}}(2.1)
$$

Here $V_{\mu}$ is the field of four-velocity, which satisfies the normalization condition

$$
g_{\mu v} V^{\mu} V^{v}=1,(2.2)
$$


$g_{\mu v}$ is the metric tensor in the Euler frame of reference,

$$
\begin{gathered}
\Sigma_{\mu v}=\nabla_{(\mu} V_{v)}-V_{(\mu} F_{v)},(2.3) \\
\Omega_{\mu v}=\nabla_{[\mu} V_{v]}-V_{[\mu} F_{v]},(2.4) \\
F_{\mu}=V^{v} \nabla_{v} V_{\mu \cdot}(2.5)
\end{gathered}
$$

Where $\Sigma_{\mu \nu}$ is the strain-rate tensor, $\Omega_{\mu v}$ is the tensor of angular velocity of rotation, and $F_{\mu}$ are the first curvature vectors of the world lines of particles of the medium. Parenthesis surrounding indices are the balancing sign and square brackets are the alternation sign. The Greek and Latin indices run values from 0 to 3 and from 1 to 3, respectively. Expansion (1) can be interpreted from the following two points of view:

1. The field of four-velocity $V_{\mu}$ is assumed to be known, for example, as a result of integration of the Euler or Navie-Stoks relativistic motion equation at a specified flat metric. The continuum characteristics $\Sigma_{\mu v}, \Omega_{\mu v}$, and $F_{\mu}$ can be obtained from formulas (2.3-2.5), and expansion (2.1) turns to identity.

2. The functions $\Sigma_{\mu v}, \Omega_{\mu v}$, and $F_{\mu}$ are assumed to be specified. Expansion (2.1) transformed into a system of differential equations with respect to $V_{v}$ and $g_{\mu v}$. The number of equations (2.1) and (2.2) exceeds the number of unknown functions, therefore, the integrability conditions must be satisfied.

$$
\frac{\partial^{2} V_{v}}{A x^{5} \partial x^{\sigma}}=\frac{\partial^{2} V_{v}}{A x^{\sigma} \partial x^{8}},(2.6)
$$

it follows from the expression that

$$
R_{\varepsilon \sigma, v}^{\mu} V_{\mu}=2 \nabla_{[\varepsilon} \Sigma_{\sigma] v}+2 \nabla_{[\varepsilon} \Omega_{\sigma] v}+2 \nabla_{[\varepsilon}\left(V_{\sigma]} F_{v}\right) \cdot(2.7)
$$

Integration of system (2.1), (2.7), where $R_{\varepsilon \sigma, V}^{\mu}$ is the curvature tensor (which is conventionally expressed in terms of the metric tensor), yields a solution to the problem on the space-time geometry, in which an NFR with a specified structure is implemented. Equations (2.7) will be referred to as structural equations for the frame of reference [4, 5, 13]. It follows from $(2.3),(2.7)$ that a rigid globally uniformly accelerated motion is shown to be realizable in the Riemannian space of a constant curature [4], [5]. The calculation in the Lagrangian accompanying NRF results in the metric

$$
d S^{2}-\exp \left(\frac{2 a_{0} y^{1}}{c^{2}}\right)\left(d y^{0}\right)^{2}-\left(d y^{1}\right)^{2}-\left(d y^{2}\right)^{2}-\left(d y^{2}\right)^{2},(2.8)
$$

where the acceleration $a_{0}$ is directed along the $y^{1}$ axis. One independent component of the curvature tensor calculated by metric (2.8) is of the form

$$
R_{10,10}=-\frac{a_{u}{ }^{2}}{c^{4}} \exp \left(2 a_{0} y^{1} / c^{2}\right) \cdot(2.9)
$$

The components of the Ricci tensor $R_{\beta \gamma}=g^{\alpha \gamma} R_{\alpha \beta \gamma \delta}$ can be written as

$$
R_{00}=-R_{10,10}, \quad R_{11}=-\frac{a_{0}^{2}}{c^{4}}, \quad R_{10}=0 .(2.10)
$$

and the scalar curvature is

$$
R=2 \frac{a_{0}^{2}}{c^{4}}
$$

\section{Theorem1}

\section{Born-rigid relativistic linearly accelerated translational motion of a continuous medium is absent in the Minkowski space.}

\section{Proving}

In agreement with formula (2.7) (structural equations), $\Omega_{\mu \nu}=0$ for the translational motion, $\Sigma_{\mu \nu}=0$ for the Born-rigid motion and the ralativistic linearly accelerated motion is characterized by the equality

$$
g_{\mu \nu} F^{\mu} F^{v}-\operatorname{const}(2.12)
$$

Under these conditions the structural equation is reduced to 
and equations (2.1-2.5) yield the relation

$$
R_{\varepsilon \sigma, v}^{\mu} V_{\mu}=\nabla_{\varepsilon}\left(V_{\sigma} F_{v}\right)-\nabla_{\sigma}\left(V_{\varepsilon} F_{v}\right) \cdot(2.13)
$$

Let us consider the convolution

$$
\nabla_{\varepsilon} V_{\sigma}=V_{\varepsilon} F_{\sigma} \cdot(2.14)
$$

$$
R_{\varepsilon \sigma, v \mu} V^{\mu} V^{\sigma}=\left(\delta_{\varepsilon}^{\sigma}-V_{\varepsilon} V^{\sigma}\right) \nabla_{\sigma} F_{V}-F_{\varepsilon} F_{V^{*}}(2.15)
$$

In turn, convlutiong with four-accelerations, we obtain In agreement with (2.12) we have

$$
R_{\varepsilon \sigma, v \mu} V^{\mu} V^{\sigma} F^{\varepsilon} F^{v}=-\left(F^{\varepsilon} F_{\varepsilon}\right)^{2}=\text { const } \neq 0 .(2.17)
$$

Thus, the convolution of the Riemann-Christoffel tensor is nonzero; therefore, the RiemannChristoffel tensor is also nonzero, and the theorem is proven.

Equation (2.7) for rigid vortex-free motions, with convolution over the first and third indices (the curvature and Ricci tensors are chosen as in [2]), using obvious identity $\nabla_{\sigma}\left(V_{\varepsilon} F^{\varepsilon}\right) \equiv 0$ and expression (2.13), yieds

$$
R_{\sigma \mu} V^{\mu}=V_{\sigma} \nabla_{\varepsilon} F^{\varepsilon},(2.18)
$$

that is equivalent

$$
\left(R_{\sigma \mu}-A g_{\sigma \mu}\right) V^{\mu}=0, A \equiv \nabla_{\varepsilon} F^{\varepsilon},(2.19)
$$

he theorem makes false the widespread opinion, according to which the Moller transformation [17] provides transition from an inertial frame of reference to a relativistic linearity accelerated NRF. This point of view is represented in the Fok monograph [18]. The Moller metric, being Born-rigid, is far from being linearly uniformly accelerated. Moller wrote hereof

$$
a\left(y^{1}\right)=\frac{a_{0}}{1+\frac{a_{0} y^{1}}{\mathrm{c}^{2}}} \cdot(2.20)
$$

It follows from the reduced formula that each Lagrange particle moves with the constant aceleration, but these accelerations are not equal one another. In regard to the known Rindler formula this is the rewriting of the Moller formula with the horizon shifted to the origin of coordinates and the transition to the dimensionless variables. An alternative to the Moller transformation is the Logunov transformation [19], which is the law of motion of charged dust (without initial velocity) in a uniform electric field. The motion of Logunov-basis particles is characterized by global linear acceleration but is not Born-rigid. Our case (2.8) satisfies the conditions of Born rigidity and global linear acceleration. However, to fulfill these conditions, one must go out beyond Minkowski space. One can to be sure that the NRF is uniformly accelerated (2.8)

$$
F^{1}=\frac{D v^{1}}{d S}=\frac{d v^{1}}{d S}+\Gamma_{00}^{1}\left(V^{0}\right)^{2}=\frac{1}{g_{00}} \Gamma_{00}^{1}=-\frac{g^{11}}{2 g_{00}} \frac{\partial g_{00}}{\partial y^{1}}=\frac{a_{0}}{c^{2}} \text { ( }
$$

The rest of the components of 4-acceleration are equal to zero. Using (2.8) we will determine the intrinsic time for a space point fixed in the NFR: $y^{2}=y^{3}=0, y^{1}=$ const. After the integration we obtain

$$
\tau_{s}=\exp \left(\frac{a_{0} y^{1}}{c^{2}}\right) \tau,(2.22)
$$

where $\tau_{s}$ is the intrinsic time for this point and $\tau$ is the world time at this point.

The latter formula was obtained by Einstein [20, p. 109]. He considered that the relation (2.22) is the exact expression for the uniformly accelerated motion. However he refused from the exact formula for the approximate one based on the Moller transformation. Einstein considered that the transition to NRF is connected with the nonlinear transformation of coordinates depending on time and do not resulting to the curvature of the NRF space-time. Other classics (for example, V. A. Fok [18], p. 280-281) had the same point of view.

\section{RELATIONSHIP BETWEEN THE STRUCTURAL AND EINSTEIN EQUATIONS}

Let us consider a continuous medium characterized by rigid vortex-free motions satisfying the Einstein equations, with some positive constant $K$ added. The Einstain equations and formula (2.19) yield

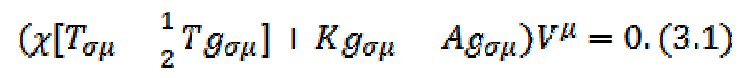


Formula is applicable for any energy-momentum tensor of matter under the conditions defined above. Macroscopic bodies are described by the known relation

$$
T_{\mu v}=(p+\varepsilon) V_{\mu} V_{v}-p g_{\mu v},(3.2)
$$

and since the four-velocity is always nonzero, we have the equation

$$
\nabla_{\mu} F^{\mu}=\frac{\chi}{2}(\varepsilon+3 p)+K, \chi=\frac{8 \pi k}{c^{4}}, F^{\mu}=V^{v} \nabla_{v} V^{\mu} .(3.3)
$$

Let us consider the one-dimensional problem in vacuum, where, in view of (2.8) $F^{1}$ in the co-moving frame of reference is given by the equality

and the metric is sought for in the form

$$
F^{1}=-\frac{g^{11}}{2 g_{00}} \frac{\partial g_{00}}{\partial y^{1}},(3.4)
$$

$$
d S^{2}=D\left(y^{1}\right)\left(d y^{0}\right)^{2}-\left(d y^{1}\right)^{2}-\left(d y^{2}\right)^{2}-\left(d y^{3}\right)^{2}
$$

When condsidering the vacuum solutions we shall remember the following.Vacuum is considered to be a highly rarefied continuous medium, composed of test particles fixed by external constraints. We assume that these particles do not form a gravitational field and do not interact with each other. Their fixation indicates that they satisfy Born's rigidity criterion. If the external constraints were absent, free particles would follow geodetic lines and be described by a synchronous frame of reference with zero four-accelerations. This system is not Born-rigid. An example is the well-known Lemaitre metric, which is derived from the Schwarzschild metric using the motion law (translation of the coordinates containing time in the nonlinear form). In the vacuum Schwarzschild metric, test particles are fixed, whereas in the Lemaitre metric they are free. Within the Schwarzschild solution, a bearing reaction force acts on a body at rest on the surface of a gravitating sphere, this force is directed along the external normal to the body and deflects the body from the geodesic. In the Newtonian mechanics the sum of forces on the surface of a gravitating sphere is zero. At the same time, the first-cuvature vector (four-acceleration) on the surface of this sphere is nonzero in GTR. Equation (3.3) for vacuum can be rewritten in the standard form

$$
\frac{1}{\sqrt{D}} \frac{d}{d y^{1}}\left(\frac{1}{2 \sqrt{D}} \frac{d D}{d y^{1}}\right)=K(3.6)
$$

The solution to this equation has the form

$$
D\left(y^{1}\right)=g_{00}=\exp \left(2 \sqrt{K} y^{1}\right)=\exp \left(2 \frac{a_{0} y^{1}}{c^{2}}\right)
$$

Here, it is taken into account that $y^{1}=0$ results in $D(0)=1$ and $F^{1}(0)=a_{0} / c^{2}$. Thus, the solution of the structural equation for the one-dimensional vacuum Einstein equation led to the metric of Bornrigid motion with global linear acceleration. At $K=0$ equation (3.6) yields the Rindler-Moller metric in flat space-time. Let us write the Einstein equation for metric (3.7).

$$
R_{\mu \nu}=K g_{\mu \nu}+\chi\left(T_{\mu \nu}-\frac{1}{2} g_{\mu v} T\right), T=T_{\sigma}^{\sigma} \cdot(3.8)
$$

Hence, the nonzero curvature coefficients in vacuum are

$$
\begin{gathered}
R_{00}=g_{00} K, R_{11}=-K=-\frac{a_{0}^{2}}{c^{4^{4}}} \\
R_{2.2}=R_{33}=-K=0 .(3.9)
\end{gathered}
$$

It follows from (3.9) that the Einstein equations for the one-dimensional stationary case are inconsistent with the exact structural equations. The situation cannot be correctd by introducing the constant $K$ into the Einstein equation. Even in vacuum the latter does not have a solution similar to that for a uniformly charged infinite plane in the STR electrodynamics. This solution is metric (2.6), which satisfies the structural equation. However, the solution to the Einstein-Maxwell equations and the structural equation considered in [21] are consistent. 
International Journal of Recent advances in Physics (IJRAP) Vol.3, No.2, May 2014

\section{Einstein Equations and Consistency Condition for Spherical AND AXIAL SYMMETRIES}

Let us consider the spherically symmetric static fields satisfying the Einstein equation, whose metric is set in the form

$$
d S^{2}=e^{v(r)}\left(d x^{0}\right)^{2}-r^{2}\left(d \theta^{2}+\sin ^{2} \theta d \phi^{2}\right)-e^{\lambda(r)} d r^{2}(4.1)
$$

\section{Theorem 2}

For a spherically symmetric static field the Einstein equations are consistent with the structural equation.

\section{Proving}

We will use the known formula [2] for metric (4.1) to calculate the Ricci tensor. We have the following expression for the desiired component $R_{0}^{0}$

$$
R_{0}^{\mathrm{C}}=e^{-\lambda}\left(\frac{v t^{\mathrm{s}}}{2}+\frac{(v t)^{2}}{4}+\frac{v t}{r}-\frac{v t \lambda}{4}\right),(4.2)
$$

where the prime indicates denote the derivative with respect to $r$. The following relations hold true in the Lagrangian (co-moving) frame of reference:

$$
V^{0}=\frac{1}{\sqrt{g_{00}}}, \quad V_{0}=\sqrt{g_{00}}, V^{k}=V_{k}=0 .(4.3)
$$

Therefore, convolution (2.15) over $V^{\sigma}$ yields the equality

After some simplifications, we have

$$
R_{0}^{0}=A=\frac{1}{2 \exp ((v+\lambda) / 2) r^{2}} \frac{d}{d r}\left(e^{(v-\lambda) / 2} r^{2} v^{\prime}\right)(4
$$

$$
e^{(v-\lambda) / 2} r^{2} v^{\prime}\left[\frac{v r^{\prime}}{v t}+\frac{v r}{2}+\frac{2}{r}-\frac{\lambda t}{2}\right]=\frac{d}{d r}\left(e^{(v-\lambda) / 2} r^{2} v^{\prime}\right)
$$

after replacement of variables,

$$
U \equiv e^{(v-\lambda) / 2} r^{2} v^{\prime}, v^{\prime}=v(4,6)
$$

and simple transformations, we find that

$$
\ln \left|\frac{n}{v r^{2}}\right|=\frac{v}{2}-\frac{\pi}{2}(4.7)
$$

This equality corresponds to identical validity of equality (4.4) at arbitrary $v(r)$ and $\lambda(r)$ and, therefore, proves the theorem. It can be shown that the well-known de Sitter, Tolman, Schwarzschild, and Reissner-Nordstrom solutions are consistent with the structure equation. The problem that is described by the equations of static gravitational field in vacuum around an immobile axially symmetric body was solved by Weyl [2]. Following [2], we have the following expression for the metric:

$$
d S^{2}=\exp (v(\rho, z))\left(d x^{0}\right)^{2}-\rho^{2} \exp (-v)(d \phi)^{2}-\exp (\gamma-v)\left((d \rho)^{2}+(d z)^{2}\right)(4.8)
$$

The consistency equation can be written as

$$
\begin{array}{r}
R_{0}^{0}=\nabla_{\mu} F^{\mu}=0,(4.9) \\
\frac{1}{2 A}\left(\frac{1}{\rho} \frac{\partial}{\partial \rho}\left(\rho \frac{\partial v}{\partial \rho}\right)+\frac{\partial^{2} v}{\partial z^{2}}\right)=0 .(4
\end{array}
$$

The equality of the expression in brackets to zero has the form of Laplace equation in cylindrical coordinates and coincides with the solution $R_{0}^{0}=0$, which was obtained in [2]. Concerning the check of the consistency of the two other equations, the structural equation in the form (4.9) does allow one to do this; here, it is necessary to use formula (2.7) for rigid vortex-free motions in the Lagrange variables. 


\section{ELECTRIC FiELD OF BOUND CHARGES}

We use the "Equivalent Situation Postulate" and mathematical formalism of NFR to find the electric field of charged conductors. Let us determine the value $a_{0}$ at the surface of the charged conductor. As it is known [22] the negative pressure force $P$ directed on external surface normal $P=1 / 2 \sigma E$ acts on the conductor surface (charged positively or negatively). Here $\sigma$ is the surface charge density, $E$ is the field strength at the conductor surface, $\sigma=N Q / S$, where $S$ is the area of the surface element, $Q$ is the charge value at the surface element, $N$ is the number of ions at the surface element for positively charged conductor or the number of electrons for negatively charged conductor. The field force created with the charges $\vec{F}=1 / 2 \vec{n} E Q$ will act on the charges at the small surface $S$. Denoting $Q / N=e$, where $e$ is the electron charge for negatively charged conductor or the ion charge for positively charged conductor we obtain the force $\vec{F}_{\theta}=1 / 2 \vec{n} E$ e acting on the electron or the ion. The force from the metall latice will act on the charges preventing the charges to leave the body surface. Thus, the field from the considered system is equivalent to the field of the charge system "moving" uniformly accelerated with the accelerations directed inside the conductor and this "acceleration" is determined with the formula

$$
\vec{a}_{0}=-\frac{1}{2} \vec{n} \frac{E s}{m},(5.1)
$$

where $m$ is the mass of electron or ion. We point out that this is the mass in the per se. It does not take into account the connection of the charges with the metallic lattice which always present. However the calculation of the effective mass is not an aim of the article. One can see from the last formula that positively charged bodies for which the numerator contains the ion charge equal to a few (or one) electron charge and the denominator contains the ion mass which is much greater then the electron mass have the "acceleration" which is very small as compared with negatively charged conductors. To find the total field of a charged conductor, one should sum the fields from charges of surface elements. The equation for a scalar potential $A_{0}$ of a point charge located at the origin of NFR coordinates (2.8) has the form

$$
\Delta A_{0}-\frac{a_{0}}{c^{2}} \frac{\partial A_{0}}{\partial y^{1}}=-4 \pi Q \exp \left(\frac{a_{0} y^{1}}{c^{2}}\right) \delta\left(y^{1}\right) \delta\left(y^{2}\right) \delta\left(y^{3}\right) \cdot(5.2)
$$

The sign $a_{0}$ is positive if the bonding force holding the charge stationary in the field coincides with the $y^{1}$ axis direction and vice versa.his equation can be obtained from Maxwell's equations written in the generally covariant form [2] similar to the case of a "given gravitational field". The details are presented in $[4,5]$. The solution of the equation results in the relation

$$
A_{0}=\frac{Q}{r} \exp \left\{-\frac{a_{0} r(1-\cos \theta)}{2 c^{2}}\right\} .(5.3)
$$

For the electric field strength $\vec{E}$ we have

$$
\vec{E}=\frac{Q}{r^{2}} \exp \left\{-\frac{a_{0} r(1-\cos \theta)}{2 c^{2}}\right\}\left[\vec{t}_{r}^{*}+\frac{a_{0} r}{2 c^{2}}\left(\frac{\vec{r}}{r}-\vec{t}\right)\right],(5.4)
$$

where $r$ is a three-dimensional (Euclidean) distance from the origin of coordinates, coinciding with the charge, to the point of observation; $\theta$ is an angle between the radius vector $\vec{r}$ and $\vec{i}, \vec{i}=\vec{a}_{0} /\left|\vec{a}_{o}\right|$. Formulas (5.2), (5.3) and (5.4) are the main for calculation of electrostatic fields of bound charges. Let us calculate the field being created by an infinite charged metallic plate of the whose thickness $h$ and determine the space-time geometry for the test charges $q$ hung on the fibers in the plate field and being immovable. On both sides of the plate the charge density will be constant and equal to $\sigma$. As in the metric (2.8) the space section is a flat a contribution to the scalar potential $A_{0}$ from the whole plate can be calculated by integrating the contribution from elementary charges $d Q$ of each of the plate side in a flat space (but not in a Riemannian space-time). We match the origin of coordinates to the plate centre by directing the $y^{1}$ axis, perpendicular to the plate, towards its upper side. After integration considering that the $a_{0}$ acceleration is directed along the communication lines holding the charge at the 
plate surface (for upper surface it is negative and for the lower surface it is positive) we find by means of (5.3) the contribution to the scalar poitential from upper plane of the plate in the upper semispace $A^{r}{ }_{0}$

$$
A^{\prime}=\frac{4 \pi \sigma c^{2}}{a_{0}} \exp \left\{-\frac{a_{0} y^{1}-h / 2}{c^{2}}\right\} .
$$

The contribution from the lower plane to the scalar potential $A^{\prime \prime}{ }_{0}$ is eqial to

$$
A^{f_{1}}{ }_{0}=\frac{4 \pi \sigma c^{2}}{a_{0}} \text {. }
$$

The contribution of both planes to the potential in the interior of the plate is equal to $\tilde{A}_{0}$

$$
\tilde{A}_{0}=\frac{8 \pi \sigma c^{2}}{a_{\mathrm{u}}} \text {. }
$$

We detremine the compound action potential in the upper semispace as the potential difference $A_{0}=A_{0}^{\prime}+A^{f_{1}}-\tilde{A}_{0}$

$$
A_{0}=-\frac{4 \pi \sigma c^{2}}{a_{0}}\left[1-\exp \left\{-\frac{a_{0} y^{1}-h / 2}{c^{2}}\right\}\right] \cdot(5.5)
$$

Developing the last formula we took into account that the metric in the lower semispace is determined from (2.8) as the reaction force acting on the charges of lower plate coincides on the direction with the $y^{1}$ axis and in the upper half-space $a_{0} \rightarrow-a_{0}$. Supposing that $h \rightarrow 0$ we find the potential in the upper half- space form the charged plane, with the plate charge density assumed equal to $\sigma_{0}=2 \sigma$

$$
A_{0}=-\frac{2 \pi \sigma_{0} c^{2}}{a_{0}}\left[1-\exp \left\{-\frac{a_{0} y^{1}}{c^{2}}\right\}\right] \cdot(5.6)
$$

The value of the field intensity of the charged plane is

$$
F_{1}=F_{01}=-\frac{\partial A_{0}}{\partial y^{1}}=2 \pi \cdot \sigma_{0} \exp \left\{-\frac{a_{0} y^{1}}{c^{2}}\right\} \cdot(5.7)
$$

We used the metric (2.8) to find the fundamental solution for the field of the unit point charge. But we are to calculate the metric itself created with the plate. Let us define the space-time metric by choosing a charged dust over the plane of like charges as the basis of a noninertial frame of reference (NFR). Let each of the particles be connected with the charged plane by a weightless and inextensible filament. As the test charges are repulsed from the plane then the filament holding the charges over the plane immovably acts on the plane direction creating the negative "acceleration". The dust particles will be mutually immovable so the strain rate tensor and the thensor of the angular velocity is equal to zero and the filament tensions are different from zero. Let the plane is infinite, the charge density on it is constant and the charge-to-mass ratios are the same for all basis particles. By definition we consider that the dust does not create the field and the field is determined only by the plane charges in accordance with (5.7). The physical situation for the dust particles in upper semispace is equivalent to the situation in some NRF "moving" down on the direction of filament tension to the plane direction as the test charges and the plane charge are similar. The space -time geometry over the charged plane has the form

$$
d S^{2}=\exp \left(-2 \frac{\left|E_{0}\right||e| y^{1}}{m c^{2}}\right)\left(d y^{0}\right)^{2}-\left(d y^{1}\right)^{2}-\left(d y^{2}\right)^{2}-\left(d y^{3}\right)^{2},(5.8)
$$

The geometry is specified with the metric (2.8) $c a_{0}=-\left|E_{0} e / m\right|$. In order to measure the field, we express the fields in terms of "physical" and tetrad components outside the charged plane. Choose the comoving tetrads for the metric so that the vector $\vec{e}_{(0)}$ should be directed along the time line, and the triad $\vec{e}_{(k)}$ - along the coordinate axes $y^{k}$ (Lames gauge [23], [24]), where the tetrad indices enclosed in brackets. The exprssion for the tetrads has the form:

$$
e_{(\alpha)}^{\mu}-\frac{\delta_{\alpha}^{\mu}}{\sqrt{\left|g_{\alpha \alpha}\right|}}, \quad e_{\mu}^{(\alpha)}-\delta_{\mu}^{\alpha} \sqrt{\left|g_{\alpha \alpha \alpha}\right|}, \quad e_{\mu}^{(0)}-V_{\mu}, \quad e_{(0)}^{\mu}-V^{\mu},(5.9)
$$

without summing over $\alpha$. For the "physical" components of the field tensor we find

$$
F_{(0)(1)}=E_{(1)}=e_{(0)}^{\mu} e_{(1)}^{v} F_{\mu \nu}=2 \pi \sigma_{0}=E_{0}=\text { const. (5.10) }
$$


From (5.10) it follows that the field of the charged plane in terms of local tetrads is the same as that conventionally considered in Cartesian coordinates. However, the space-time geometry being due to an electrostatic field proved to be pseudo-Riemannian and not pseudo-Euclidean.

The energy density of the electromagnetic field corresponds to the $T_{(0)(0)}=T^{(0)(0)}=T_{(0)}^{(0)}$ components of the energy momentum tensor of the electromagnetic field which in curvilinear coordinates has the form [1]

As the space-time out-of-plane is pseudo-riemannian then when determining the energy density (unlike STR ) one must to select between $T_{00}, T^{00}{ }_{H} T_{0}^{0}$ components of the energy momentum tensor of electromagnetic field. In our case $T_{(0)(0)}=T^{(0)(0)}=T_{(0)}^{(0)}$ components of energy momentum tensor. Energy momentum tensor of electromagnetic field in curvilinear coordinates has the form [2]

$$
T_{\mu \nu}=\frac{1}{4 \pi}\left(-F_{\mu \beta} F_{v}^{\beta}+\frac{1}{4} F_{\beta \gamma} F^{\beta \gamma} g_{\mu \nu}\right),(5.11)
$$

where the tetrad components of the tensor are calculated by the rule

$$
T_{(\mu)(v)}=e_{(\mu)}^{\alpha} e_{(v)}^{\beta} T_{\alpha \beta \cdot}(5.12)
$$

The field energy may be calculated according [24] by the formula

$$
W=\int \sqrt{-g} T^{\mu \nu} V_{v} d S_{\mu},(5.13)
$$

where $g$ is the metric tensor determinant, $d S_{\mu}-V_{\mu} d V \cdot d S_{\mu}$ is a geometrical object equal to the product of an element of the hypersurface, orthogonal to the basis world lines and based on three infinitesimally small displacements, by a unit vector of the normal ( i. e. the 4-velocity $V_{\mu}$ ).

Since we consider the electromagnetic field energy-momentum tensor outside charges creating the field, the relation should be valid

$$
\nabla_{\mu} I^{\mu \nu}=0 .(5.14)
$$

In a flat space-time in Galilean coordinates this relation is the electromagnetic field energy-momentum conservation law. In Riemannian spacetime this relation, generally, is not a conservation law at all, since a covariant derivative of the energy-momentum tensor enters in it instead of a partial one.

While calculating the energy in (5.13), we, in fact, use not the "conservation law" (24), but the real conservation law resembling the charge conservation law.

This follows from the equality

$$
\nabla_{\mu}\left(T^{\mu \nu} V_{v}\right)=V_{v} \nabla_{\mu} T^{\mu v}+T^{\mu v} \nabla_{\mu} V_{v}=T^{\mu v} V_{\mu} F_{v}=0 .(5.15)
$$

In deriving (5.15) we have allowered for the congruence of world lines of FR basis particles is being irrotational and rigid, the vectors $V_{\mu}$ and $F_{\mu}$ being orthogonal, and the convolution of the antisymmetric field tensor $F_{y \nu}$ with the product of first curvature vectors being equal to zero.

Relation (5.15) may be rewritten in the form

$$
\nabla_{\mu} T^{\mu(0)}=\frac{1}{\sqrt{-g}} \frac{\partial}{\partial y^{\mu}}\left(\sqrt{-g} T^{\mu(0)}\right)=0 .(5.16)
$$

We integrate expression (5.16) over an invariant 4-volume

$$
\int \sqrt{-g} \nabla_{\mu} T^{\mu(0)} d^{4} y=\int \frac{\partial}{\partial y^{l}}\left(\sqrt{-g} T^{\mu(0)}\right) d^{4} y=0 .(5.17)
$$

Using the Gauss theorem and assuming that on the "lateral" timelike hypersurface, enclosing a spatial volume, the integral vanishes (which is valid in problems with charges located in a finite volume), we obtain

$$
\oint \sqrt{-g} T^{\mu(0)} d S_{\mu}=\int_{V_{1}} \sqrt{-g} T^{(0)(0)} d^{3} y-\int_{V_{2}} \sqrt{-g} T^{(0)(0)} d^{3} y=0 .(5.18)
$$

Whence the conservation law of the "charge" - type quantity follows

$$
\int_{V_{1}} \sqrt{-g} T^{(0)(0)} d^{3} y=\int_{V_{2}} \sqrt{-g} T^{(0)(0)} d^{3} y,(5.19)
$$

wherein $V_{1}$ and $V_{2}$ are the three-dimensional volumes occupied by the field at different instants.

Calculate the field energy of a negatively charged plane. In the standard calculation the plane field energy of the field strength $E$, confined within a cylinder with the base area $S$ and height $h$ on each side of the plane with the elements of the cylinder perpendicular to the plane, is evidently 


$$
W_{0}=\frac{E^{2}}{8 \pi} 2 h S=\sigma_{0} Q h, \quad Q=\sigma_{0} S \cdot(5.20)
$$

For $h \rightarrow \infty, W_{0} \rightarrow \infty$.

In this case the field energy in the above-mentioned volume

$$
W=\int \sqrt{-g} I^{(0)(0)} d V=2 \frac{E^{2} s c^{2}}{B \pi a_{0}}\left(1-\exp \left(-\frac{a_{0} h}{c^{2}}\right)\right),(5.21)
$$

where $E=2 \pi o_{0}$.

It is evident that at small distances $h$ from the plane, i. e. for

$$
\frac{a_{0} h}{c^{2}} \ll 1
$$

the field energy in volume calculated according to (5.21) coincides with the classical expression (5.20). However, although in the classical consideration for $h \rightarrow \infty, W_{0} \rightarrow \infty$, in this case the field energy inside an infinitely long cylinder remains a finite quantity defined by the equality

$$
W=\int \sqrt{-g} T^{(0)(0)} d V=2 \frac{E^{2} s c^{2}}{\mathrm{~B} \pi a_{0}}=\frac{E S c^{2} m}{2 \pi \varepsilon}=\frac{Q m c^{2}}{e}=N m c^{2} \text {. (5.22) }
$$

Thus:

The energy of the electric field inside a long cylinder proved to be equal to the rest energy of $N$ electrons located on a charged surface of the area $S$ inside the cylinder. The charge $Q$ of an area element does not enter in this energy.

Taking into account that the space section for the metric (2.8) is a plane and the acceleration is negative we find the expression for the energy momentum tensor in co-moving tetrads

$$
T_{(0)(0)}=\frac{E^{2}}{8 \pi}, \quad T_{(1)(1)}=-\frac{E^{2}}{8 \pi}, \quad T_{(2)(2)}=T_{(3)(3)}=\frac{E^{2}}{8 \pi} \text {. (5.23) }
$$

The last relation in the local tetrads coincides with the energy momentum tensor of constant uniform field in the IRF of the Minkowski space in the Galilean coordinates.

\section{Centrally Symmetric Electrostatic Field}

Consider an electrostatic field having a central symmetry. This field may be created by a charged spherical body or a point charge. Consider a charged conducting ball of radius $R$ and charge $Q$. It is necessary to find the electric field strength $\vec{E}$ and determine the space-time geometry outside the ball. By analogy with the plane field we have

$$
d A_{0}=\frac{d Q}{r r} \exp \left\{-\frac{a_{0} r r(1-\cos \theta)}{2 c^{2}}\right\},(6.1)
$$

where $r^{t}$ is a three-dimensional (Euclidean) distance from the charge $d Q$ to the point of observation, $\theta$ is an angle between the radius vector $\vec{r}^{\prime}$ and $\vec{i}, \vec{i}-\vec{\alpha}_{0} /\left|\vec{\alpha}_{0}\right| . \vec{i}$ is directed to the sphere centre for an element $d Q$ of the charge. The value of the "acceleration" $a_{0}$ for charges of the negatively charged sphere (electrons) is calculated by the formula

$$
a_{0}=\frac{\Theta E}{2 m},(6.2)
$$

Here $e$ is the charge value, $m$ is the electron mass, $E$ is a field strength on the sphere surface. For the ball charged positively the "relativistic" effect will be much less pronounced than for the negatively charged one, because the mass $m$ of the positive ion exceeding the electron mass. Hence the field from a positively charged ball almost coincides with the classical one, and for a negatively charged ball the "relativistic" corrections may prove to be considerable.

In accordance with (2.8) each electron at the sphere surface belongs to the tangent plane space but riemannian space-time. Therefore the intergation operation on the sphere occurs in the plane space and it is correct. Integrating (6.1), we obtain

$$
A_{0}=\frac{Q \exp \left(-\tau_{2}^{2}\right) \sqrt{\pi} t}{4 R \zeta}[\Phi(i \zeta(1-2 R / r))-\Phi(i \zeta)], \quad \zeta=\frac{r}{R} \sqrt{\frac{\theta Q}{8 m c^{2} R}},(6.3)
$$


$r$ is a distance from the ball centre to the point of observation, $\Phi(\zeta)$ is the probability integral

$$
\Phi(i \zeta)-\frac{2 t}{\sqrt{\pi}} \int_{0}^{\zeta} \exp \left(t^{2}\right) d t
$$

On the other hand the set of electrons on the sphere surface does not belong to the congruence of world lines of the NFR basis (2.8) but are included in the set of world lines belonging to the Lagrangian comoving spherically symmetric NFR with the metric

$$
d S^{2}=\exp (v)\left(d y^{0}\right)^{2}-r^{2}\left(d \theta^{2}+\sin ^{2} \theta d \phi^{2}\right)-\exp (\lambda)(d r)^{2} \text { (6.4) }
$$

The functions $v(r)$ and $\lambda(r)$ need to be determined. To find them, we resort to a solution to Maxwell's spherically symmetric static equations, using metric (6.4), similar to the notation of the equations of electrodynamics in a "given gravitational field" [2]. Then compare the solution obtained with the expression for the field derived from (6.3).

For the radial component "induction" $D^{1}$ differed from zero we have the equation

$$
\frac{1}{\sqrt{\gamma}} \frac{\partial}{\partial r}\left(r^{2} \sin \theta \exp (\lambda / 2) D^{1}\right)=0,(6.5)
$$

the solution of which will be

$$
D^{1}=\frac{Q}{r^{2}} \exp (-\lambda / 2) \cdot(6.6)
$$

There are the known relations between the "induction" $D^{1}$, field strength $E^{1}$ and the field tensor component $F_{01}[2]$

$$
D_{1}=\gamma_{11} D^{1}=\frac{Q}{r^{2}} \exp (\lambda / 2)=\frac{1}{\sqrt{g_{00}}} E_{1}, \quad E_{1}=F_{01}, \quad \gamma_{k l}=-g_{k l}(6.7)
$$

where $\gamma_{k l}$ is the space metric tensor with the determinant equal to $\gamma$.

In accordance with (6.3), (6.4) the electromagnetic field tensor $F_{\mu v}$ has the components $F_{01}=-F_{10}$ differed from zero

$$
\begin{gathered}
F_{\mathrm{U} 1}=\frac{Q \exp \left(-\zeta^{2}\right) \sqrt{\pi}}{4 r^{2} \delta}\left\{[\Phi(i \zeta-2 i \delta)-\Phi(i \zeta)]\left(1+2 \zeta^{2}\right) i-\right. \\
\left.\frac{2 \zeta \exp \left(\zeta^{2}\right)}{\sqrt{\pi}}[1-\exp (-4 \delta(\zeta-\delta))]\right\}, \quad \delta=\sqrt{\frac{a Q}{8 m c^{2} R}} \cdot(6.8)
\end{gathered}
$$

Equating (6.8) to expression $F_{01}$ from (6.7), we find the constraint equation on the functions $v(r)$ and $\lambda(r)$

$$
\begin{gathered}
\exp \left(\frac{v+\lambda}{2}\right)=\frac{\exp \left(-\zeta^{2}\right) \sqrt{\pi}}{4 \delta}\{[\Phi(i \zeta-2 i \delta) \\
\left.-\Phi(i \zeta)]\left(1+2 \zeta^{2}\right) i-\frac{2 \zeta \exp \left(\zeta^{2}\right)}{\sqrt{\pi}}[1-\exp (-4 \delta(\zeta-\delta))]\right\} .(6.9)
\end{gathered}
$$

To find the second equation connecting these functions, we consider a force, being due to the field, acting on a test charge $q$ fixed at the point $r$ from the ball centre. Let the mass of the test charge be $m_{0}$. Then the first curvature vector $F^{1}$ of the world line of this charge can be found from the relation

$$
F^{1}=\frac{1}{2} \frac{d v}{d r} \exp (-\lambda)
$$

and from the force acting on the charge due to the constraint holding it immovable in the field. This force is numerically equal to the force due to the field and is opposite to it in sign.

$$
F^{1}=\frac{1}{2} \frac{d v}{d r} \exp (-\lambda)=-\frac{q}{m_{0} c^{2}} F^{10} V_{0}=-\frac{Q q}{m_{0} c^{2} r^{2}} \exp (-\lambda / 2) \text {. (6.10) }
$$

As a result, we find

$$
\begin{gathered}
\exp (v / 2)=-\frac{q}{m_{0} c^{2}} \int F_{01} d r \\
=-\frac{Q q \sqrt{\pi}}{4 R m_{0} c^{2}} \int\left\{\exp \left(-\zeta^{2}\right)[\Phi(i \zeta-2 i \delta)-\Phi(i \zeta)]\left(1 / \zeta^{2}+2\right) i\right. \\
\left.-\frac{2}{\zeta \sqrt{\pi}}[1-\exp (-4 \delta(\zeta-\delta))]\right\} d \zeta+C_{1} \cdot(6.11)
\end{gathered}
$$

We determine the integration constant $C_{1}$ from the requirement of space being Euclidean at infinity. Performing the integration in (6.11) analytically presents no problems, since by definition 


$$
F_{01}=-\frac{\partial A_{0}}{\partial r^{2}}
$$

where $A_{0}$ is determined from (6.3). As a result, we obtain

$$
\begin{gathered}
\exp (v / 2)=1+\frac{Q q \exp \left(-\zeta^{2}\right) \sqrt{\pi} i}{m_{0} c^{2} 4 r \varepsilon}\lceil\Phi(i \zeta-2 i \delta)-\Phi(i \zeta)\rceil \\
=1+\frac{q A_{2}}{m_{0} c^{2}} .(6.12)
\end{gathered}
$$

From (6.10) and (6.12) we have

$$
\begin{aligned}
& \exp \left(\frac{\dot{H}}{2}\right)=\frac{\left.\frac{\operatorname{axp}\left(-\zeta^{2}\right) \sqrt{\pi}}{4 \delta}[[\Phi(t \zeta-2 t \delta)]-\Phi(t \zeta)]\left(1+2 \zeta^{2}\right) t\right]}{1+\frac{0 q \exp \left(\zeta^{2}\right) \sqrt{\pi} t}{m_{0} c^{2} 4 r \delta}\left[\Phi\left(i \zeta_{2}-2 i \delta\right)-\Phi(i \zeta)\right]} \\
& -\frac{\frac{\zeta}{2 \delta}[1-\exp (-4 \delta(\zeta-\delta))]}{1+\frac{\left.Q q e x p l-\zeta^{2}\right) \sqrt{n} t}{m_{0} c^{2} 4 r \delta}[\Phi(i \zeta-2 i \delta)-\Phi(i \zeta)]}=-\frac{\frac{r^{2} \partial A_{0}}{Q \partial \partial r}}{1+\frac{q A_{0}}{m_{0} c^{2}}} .
\end{aligned}
$$

Analyze (6.12), (6.13) expanding the expressions as a series in a dimensionless parameter $\delta$.

For an electron with the classical radius $e^{2} / m c^{2}, \delta=0.35355$, for a proton $\delta=0.015$. The expansion in terms of $\delta$ with retaining terms proportional to the first power of $\delta$ (in the numerator of (6.13) we expand into a series retaining terms with $\delta^{2}$ ) leads to the relation

$$
\exp (v)=\left(1+\frac{Q q}{r m_{0} c^{2}}\right)^{2}=\exp (-\lambda)
$$

In formula (6.14) the charges $Q$ and $q$ can be of equal as well as opposite signs. As distinct from GR, the space-time metric depends both on the charge $Q$ creating a field and on the value of a test charge $q$ . If as the test charges one chooses equal charges whose Coulomb's repulsion force is exactly equal to Newton's attraction force, then such particles will be classically noninteracting. If, besides, one equates Newton's gravitational force between charges on the sphere to its Coulomb's electrostatic repulsion, then evident relations $q^{2} / m_{0}^{2}=k, Q^{2} / M^{2}=k$ will be valid, where $M$ is a mass of charges $Q, k$ is the gravitational constant. (The solution is presented in SGSE units). Metric (6.14) in this case coincides with an exact electrovacuum spherically symmetric consistent solution to Einstein's and Maxwell's equations and is called Reissner-Nordström's metric.

Estimate the value of $\delta$ at which an approximate solution (i.e. Reissner-Nordström's) differs least from the exact one (6.12), (6.13).

Choose the electron charge $e$ as the least charge. Then from the equality $e^{2} / M^{2}=k$ we find $M=1.86 \cdot 10^{-6} \mathrm{~g}$. The Markov "maximons" have such property [25]. Considering the particle to be spherical and assuming its density to be equal to that of nuclear matter $\rho=2.7 \cdot 10^{14} \mathrm{~g} / \mathrm{cm}^{3}$, we find $\delta=2.5 \cdot 10^{-5}$ in accordance with (6.8). Since we have obtained Reissner-Nordström's solution as an expansion in terms of $\delta$, it almost coincides with the exact one for the last example.

Thus, for the particles having the electron charge in which the coulomb repulsive force is compensated with the newton gravitational force the Raisner-Nordstream solution is correct. In the EinsteinMaxwell theory such contingencies are absent. At last a formal substitution of coupling constants in (6.14) $Q q \rightarrow-k M m_{0}$ in first approximation results in Schwarzschild's metric.

Calculate the capacitance $C$ of a charged metallic ball of radius $R$. When considering classically, we use the formula $C=R$.

Calculate the capacitance by the formula

$$
C=Q^{2} / 2 W \cdot(6.15)
$$

The energy density $\rho$ of the electromagnetic field corresponds to the $T_{(0)(0)}=T^{(0)(0)}=T_{(0)}^{(0)}$ components of the energy-momentum tensor. Obviously

$$
\rho=T_{(0)(0)}=T_{\mu \nu} V^{\mu} V^{\nu} .
$$


or

$$
\rho=\frac{Q^{2}}{8 \pi r^{4}},(6.16)
$$

which coincides with the expression for the field energy density outside the charged sphere in Minkowski space.

To find the total fields energy outside the charged ball we have

$$
W=\frac{Q^{2}}{2} \int_{R}^{\infty} \frac{\exp \left(\frac{\lambda+v}{2}\right)}{r^{2}} d r=\frac{A_{0}(R) Q}{2},(6.17)
$$

where $A_{0}(R)$ is determined from (36) at $r=R$.

As a result, we obtain for the capacitance $C$

$$
C=R\left[\frac{2 t \delta}{\sqrt{\pi} \exp \left(-\delta^{2}\right) \Phi(t \delta)}\right]
$$

Considering that $\delta$ is a small quantity, we find from (51)

$$
C=R\left[1+\frac{2}{3} \delta^{2}\right],(6.19)
$$

One may to determine the ball capacitance conventionally by the formula

$$
C-\frac{Q}{A_{0}} \cdot(6.20)
$$

where $A_{0}$ is calculated from (6.3) at $r-R$.

Thus, both formulae proved to be equivalent as well as in the classical consideration.

As known [2], in classical relativistic mechanics finite dimensions cannot be assigned to elementary particles, and they should be considered to be pointlike. On the other hand the charged particle under such consideration has an infinite proper energy as well as a mass. The physical senselessness of this result calls for the fundamental principles of electrodynamics itself to be bounded by certain limits.

We prove that in the model proposed the above-mentioned difficulty is absent, and the proper energy of a negative point charge $Q$ containing $N$ electrons corresponds to the quantity $W=2 N m c^{2}$.

To prove it, we use expressions (6.17) and (6.3) at $R \rightarrow 0$. Assuming in (6.17) $Q=\mathrm{Ne}$ and making use of the well-known asymptotic formula [26]

$$
\sqrt{\pi} z \exp \left(z^{2}\right)(1-\Phi(z)): 1+\sum_{m=1}^{\infty}(-1)^{m} \frac{1 \cdot 3 \ldots(2 m-1)}{\left(2 z^{2}\right)^{m}},(6.21)
$$

we find for $z=\delta$ ? 1 the expression

$$
\begin{gathered}
A_{0}(R) \approx \frac{Q}{2 R \delta^{2}}\left(1+\frac{1}{2 \delta^{2}}\right)=\frac{4 m c^{2}}{e}\left(1+\frac{4 m c^{2} R}{\varepsilon Q}\right) \\
W=\frac{Q A_{0}}{2}=2 N m c^{2}\left(1+\frac{4 m c^{2} R}{e^{2} N}\right) .
\end{gathered}
$$

Whence for $R \rightarrow 0$ we have

$$
W=2 N m c^{2} \cdot(6.22)
$$

The quantity of energy of a point particle proved to be independent of the sign and value of the charge. From (6.22) it follows that the field energy of a negatively charged particle with the charge $Q-N o$ is determined by the total rest energy of its $2 N$ electron masses. In particular, for one electron "smeared" over a sphere of radius the $R \rightarrow 0$, the proper field energy coincides with its redoubled rest energy $W=2 m c^{2}$.The nature of the forces preventing from the electron explosion as a result of the action of the coulomb forces is unknown. The solution of this problem can be obtained only in the general theory of elementary particles. As it has been pointed out [1] in 1906 Poincare"introduced the surface pressure of the unknown origin which must to act on the electron all round as the uniformly stretched film".

This film is eqivalent to our model of elastic threads fixed at the cenrte and holding the elements of the electron charge from the recession stipulated for the Coulomb forces. Apparently in this Poincare pressure (or the elastic strain forces) the part of the electron rest energy must to hide. Therefore electromagnetic electron mass is greater than the rest mass. The part of electromagnetic mass is responsible for its stability. 
Similar situation takes place in GR when considering the total energy of the matter and the constant gravitational field (i. e. the total mass of the body) expressed via the energy momentum tensor of only one matter [2] .First this problem has been considered by P. Tolmen. Eliminating the intermediate steps which are made in detail in [2] we present the result

$$
P^{0}=m c=\frac{1}{c} \int\left(T_{0}^{0}-T_{1}^{1}-T_{2}^{2}-T_{3}^{3}\right) \sqrt{-g} d V .
$$

Here $P^{0}$ is the time components of 4 - pulse, $d V$ is the three-dimensional (Euclidean) volume element, $g$ is the four-dimensional determinant of the metric tensor.

If the energy momentum tensor of electromagnetic field has been selected as the source in the Einstein equations then from the equalities

$$
T_{\mu}^{\mu}-0, T^{(0)(0)}-T_{0}^{0},
$$

following from the tetrades in the form (5.9) using the metric (6.4) and the accompanying conditions in the form

we find

$$
V^{k}=V_{k}=0, \quad V^{0}=\left(g_{00}\right)^{-1 / 2}, \quad V_{0}=\left(g_{00}\right)^{1 / 2},
$$

$$
P^{0}=m c=\frac{2}{c} \int T^{(0)(0)} \sqrt{-g} d V .
$$

The $P^{0}$ value differes from the $W$ field energy by the $2 / c$ multiplier. If the $1 / c$ multiplier is natural, then the presence of the energy doubling is connected with both the energy of electric field and the gravitational field connected by means of the Einstein equations.

In our case the field of the bonding forces (the Poincare pressure) plays the role of the gravitational field. The bonding forces prevent to the Coulomb repulsion forces and keep the rigidity of the charged particle. Therefore the doubling of the energy occurs in (6.22).

Although the potential $A_{0}$ and energy $W$ of the point charge field are finite, however the expression for the Coulomb law proves to be true in the tetrads for all range of radius values. For the tetrad components differed from zero the expression follows

$$
F_{(0)(1)}=\exp \left(\begin{array}{c}
\lambda+\mu \\
2
\end{array}\right) F_{01}=\underset{r^{2}}{Q}
$$

which exactly coincides with the classical expression for the field strength of the point charge for any $v$ and $\lambda$.

\section{AXIALly SYMMETRIC FIELD}

Consider an axially symmetric field being created by an infinitely long charged metallic cylinder. We match the $z$ axis to the cylinder one, having chosen the origin of coordinates at the cylinder centre. The calculation of the field will be performed in a cylindrical system of coordinates, using formula (6.1) to find the potential, where $r^{\prime}$ is the three-dimensional (Euclidean) distance from the charge $d Q$ to the observation point; $\theta$ is the angle between the radius vector $\vec{r}$ and $\vec{i}, \vec{i}=\vec{a}_{0} /\left|\vec{a}_{0}\right| . \vec{i}$ for each element of the charge $d Q$ is directed towards the centre of the cylinder perpendicular to its surface. The quantity of "acceleration" $a_{0}$ for charges of a negatively charged cylindrical surface (electrons) is calculated by formula (6.2). From geometrical considerations it is easy to obtain a formula relating the quantities $r^{\prime}$ and $\theta$ to the radial $\rho$ and angular $\phi$ cylindrical coordinates.

$$
r^{\prime} \cos \theta=R-\rho \cos \phi \cdot(7.1)
$$

The charge element $d Q$ on the cylinder surface is representable in the form

$$
d Q=\sigma R d \phi d z=\frac{\gamma}{2 \pi} d \phi d z,(7.2)
$$

where $\sigma$ and $\gamma$ are the surface and linear densities of charges. For the potential of the charged element of the conductive cylinder surface we obtain the expression

$$
d A_{0}=\frac{\gamma}{2 \pi r r} \exp \left\{-\frac{a_{0} r r(1 \mp \cos \theta)}{2 c^{2}}\right\} d \phi d z,(7.3)
$$

In the last formula when selecting the exponent sign we take into account two possibilities. The upper sign means that the charges are located on the cylinder surface with the "acceleration" directed to the cylinder centre (for example, at the interior electrode of the cylindrical capacitor). The bottom sign 
corresponds to the charges locating at the interior surface of the cylindrical tube (for example, at the external electrode of the cylindrical capacitor). Apparently that the charge "accelerations" always directed inside the metal at the internal and the external capacitor plates have the diametrically opposite directions. The sign selection in (7.3) is connected with this circumstance.From (7.3) we have $A 0=2 \gamma \pi \varepsilon \pm p R 0 \pi \theta \mp p \rho \cos \phi\left(\rho 2+\infty \theta-p r^{\prime} r^{\prime} 2-\rho 22 d r^{L}\right) d \phi,(7,4)$

where the designations are introduced

$$
p=\frac{a_{0}}{2 i^{2}}, \quad \rho_{2}=\sqrt{\rho^{2}+R^{2}-2 \rho R \cos \phi} \cdot(7.5)
$$

The calculation of the inner integral results in the relation

$$
A_{0}=\frac{2 \gamma}{\pi} e^{ \pm p R} \int_{0}^{\pi} e^{\mp p \rho \cos \phi} K_{0}\left(\rho_{2} p\right) d \phi(7.6)
$$

$K_{0}$ are the cylinder Bessel functions in the generally accepted designations [26].

To calculate the integral (7.6) we use a known "either-or theorem" for the cylinder functions [27]. Preliminarily we shall introduced the following designations .

$$
R_{1}=R p, \quad R_{2}=\rho_{2} p, \quad \rho_{1}=R_{1} \frac{\rho}{R}=\rho p .(7.7)
$$

Specifically for the cylinder Hankel functions $H_{0}^{(1)}\left(i R_{2}\right)$ the relation is true

$$
H_{0}^{(1)}\left(i R_{2}\right)=J_{0}\left(i R_{1}\right) H_{0}^{(1)}\left(i \rho_{1}\right)+2 \sum_{k=1}^{\infty} J_{k}\left(i R_{1}\right) H_{k}^{(1)}\left(i \rho_{1}\right) \cos k \phi \cdot(7.8)
$$

The last formula is valid provided that $R_{1}<\rho_{1}$. Taking into account the connection between the Hankel functions and the modified Bessel functions

$$
K_{v}(z)=\frac{\pi i}{2} \exp \left(\frac{\pi i v}{2}\right) H_{v}^{(1)}(i z), \quad J_{k}(i z)=i^{k} I_{k}(z),(7.9)
$$

we find

$$
K_{0}\left(R_{2}\right)=I_{0}\left(R_{1}\right) K_{0}\left(\rho_{1}\right)+2 \sum_{k=1}^{\infty} I_{k}\left(R_{1}\right) K_{k}\left(\rho_{1}\right) \cos k \phi .(7.10)
$$

Taking into account the last formula we calculate the integral (7.6)

$$
A_{0}=2 \gamma e^{ \pm R_{1}}\left[I_{0}\left(R_{1}\right) I_{0}\left(\rho_{1}\right) K_{0}\left(\rho_{1}\right)+2 \sum_{k=1}^{\infty}(\mp 1)^{k} I_{k}\left(R_{1}\right) I_{k}\left(\rho_{1}\right) K_{k}\left(\rho_{1}\right)\right] .(7.11)
$$

Obtained formula is valid when $\rho>R$, i.e. for the potential outside the cylinder. For the potential in the interior of the cylinder at $\rho<R$ the formula takes the form

$$
A_{0}=2 \gamma e^{ \pm R_{1}}\left[I_{0}\left(\rho_{1}\right) I_{0}\left(\rho_{1}\right) K_{0}\left(R_{1}\right)+2 \sum_{k=1}^{\infty}(\mp 1)^{k} I_{k}\left(\rho_{1}\right) I_{k}\left(\rho_{1}\right) K_{k}\left(R_{1}\right)\right] \cdot(7.12)
$$

At the cylinder boundary at $\rho=R$ both formulas apparently coincide. Let us obtain the potential (7.12) at the great distances from the cylinder, i. e. supposing that $\rho$ ? $R$. Using the known asymptotic expansion [26] applicable for the large $z$

$$
I_{v}(z) K_{v}(z): \frac{1}{2 z},(7.13)
$$

and also the known summation formula

$$
2 \sum_{k=1}^{\infty}(\mp 1)^{k} I_{k}(z)-e^{\mp z}-I_{0}(z),(7.14)
$$

we find

$$
A_{0}\left(\rho_{1} ? 1\right)=\frac{\gamma}{\rho_{1}}=\frac{z \gamma c^{2}}{a_{0} \rho}=\frac{z m c^{2} R}{\Theta \rho},(7.15)
$$

where $m$ is the electron mass if the cylinder is charged negatively or the nuclear mass of the metal, at positive charge, $e$ is the electron charge having the negative value at negative charge of the cylinder and the positive value (equal to the electron charge) at positive cylinder charge. When $\rho \rightarrow \infty$ the potential tents to zero. It does not logarithmically diverge as in classical representation. Thus, the potential behaviour at infinity sharply differes from the classical analog that eventually results in the elimination of the divergence of electromagnetic field energy.Formula (7.15) is applied not only for the great distances from the wire. $\rho_{1}, R_{1}$ values can be very large and on the cylinder surface with a small radius if the charges densities at the wire are great. For the great charge densities the potential at the surface of the cylinder with finite radius has the form

$$
A_{0}(R)=\frac{\gamma}{R_{1}}=\frac{2 \gamma c^{2}}{a_{0} R}=\frac{2 m c^{2}}{e} \cdot(7.16)
$$


The electric field energy outside the cylinder shell can be calculated in accordance with the general formula (6.17) and it results in the relation

$$
W=N m c^{2} \cdot(7.17)
$$

The value of the field energy outside the conducting shell when great charge density turns out independent on the sign and the charge value. It follows form the presented formula that the field energy of negatively charged shell with the charge $Q=N e$ on the length $h$ is determined by the total rest energy of its $N$ electron masses. For positively charged shell the nuclear mass plays the mass role.

In the classical representation the field energy outside the shell tends to infinity. For the cylinder and the plane fields unlike the sphere field the doubling of the energy does not occur. In detail these prblems are considered in $[4,5]$. Let us investigate the field behaviour inside the cylindrical shell. In the classical representation the potential inside the shell is constant and it is equal to the shell potential and the electric field strength is equal to zero. In the field of bound charges it is not the case. As it follows from the solution (7.12) the potential inside the shell is not a constant value. Therefore the field strength is differed from zero. It is calculated in accordane with the formula

$$
\begin{gathered}
F_{01}=-4 \gamma p e^{ \pm R_{1}}\left[I_{0}\left(\rho_{1}\right) I_{1}\left(\rho_{1}\right) K_{0}\left(R_{1}\right)+\right. \\
\left.+2 \sum_{k=1}^{\infty}(\mp 1)^{k} K_{k}\left(R_{1}\right) I_{k}\left(\rho_{1}\right)\left(I_{k-1}\left(\rho_{1}\right)-\frac{k}{\rho_{1}} I_{k}\left(\rho_{1}\right)\right)\right] \cdot(7.18)
\end{gathered}
$$

Let us investigate the potential behaviour inside the shell for the case of the low fields. Using the known expansions for the small values of argument [26]

$$
I_{k}\left(\rho_{1}\right): \frac{\rho_{1}^{k}}{2^{k} \Gamma(k+1)}, \quad K_{k}\left(R_{1}\right): \frac{1}{2} \Gamma(k) \frac{2^{k}}{R_{1}^{k}},(7.19)
$$

where $\Gamma(k)$ is the gamma function, after summation we obtain the expression

$$
\begin{aligned}
& \left.F_{01}=\underset{c^{2}}{2 \gamma a_{0}} e^{+R_{1}}\left[\begin{array}{c}
\rho_{1} \\
2
\end{array}\right] n R_{1} \mid{ }_{\rho_{1}}^{1} \sum_{k=1}^{\infty}(\mathrm{T} 1)^{k}{ }_{k !}^{1}\left(\rho_{2 R_{1}}^{\rho_{1}^{2}}\right)^{k}\right]= \\
& \left.=\left.c^{2} a_{0} e^{+R_{1}}\left[\begin{array}{c}
\rho_{1} \\
2
\end{array}\right] R_{1}\right|_{\rho_{1}} ^{1}\left(\exp \left(\mathrm{T}_{2 R_{1}}^{\rho_{1}^{2}}\right) \quad 1\right)\right] \cdot(7.20)
\end{aligned}
$$

Limiting by the terms containing the $1 / c^{2}$ multiplier we obtain the expression for the value of electric field strength

$$
F_{01}--\frac{\partial A_{0}}{\partial \rho}-E(\rho)- \pm E_{0} \frac{a_{0} \rho}{2 c^{2}}- \pm E_{0} \frac{E_{0} e \rho}{4 m c^{2}}, \quad E_{0}-\frac{2 \gamma}{R} \cdot(7.21) \mathrm{z}
$$

Here $E_{0}$ is the classical value of the field strength at the surface of the charged conducting cylinder. It follows from the formula that unlike the classical case the field inside the cavity is not equal to zero. The field linearly increases with the rise of the distance to the centre however unlike the potential the field strength at the cylinder boundary has a discontinuity.

Let us find the space-time metric outside the cylinder. To simplify we pass from the cylinder to the charged filament supposing that $R \rightarrow 0$ and considering that $\gamma$ is the constant finite quantity. As a result for the $A_{0}$ potential from [66] we obtain the expression

$$
\begin{gathered}
A_{0}=2 \gamma I_{0}(\alpha) K_{0}(\alpha), \quad \alpha=\frac{\rho a_{0}}{2 c^{2}}=\frac{\rho \rho E_{0}}{4 m c^{2}},(7.22) \\
d S^{2}=\exp (v)\left(d y^{0}\right)^{2}-\exp (\lambda) d \rho^{2}-\rho^{2} d \phi^{2}-d z^{2},(7.23)
\end{gathered}
$$

where $v$ and $\lambda$ depend only on $\rho . v(\rho)$ and $\lambda(\rho)$ finctions have to be determined. To find them we use the solution of the cylindrically symmetrical static Maxwell equations with the utilization of the metric (7.23) similar to the electrodynamic equations in the "specified gravitational field" [2]. Then we shall compare the obtained solution and the expression for the field obtained from (7.22). For the radial component $D^{1}$ "induction" differed from zero we have the equation

the solution of it will be

$$
{ }_{\sqrt{\gamma} \partial \rho}^{1 \partial}\left(\rho \exp (\lambda / 2) D^{1}\right)=0,(7.24)
$$


International Journal of Recent advances in Physics (IJRAP) Vol.3, No.2, May 2014

$$
D^{1}-\frac{2 \gamma}{\rho} \exp (-\lambda / 2) \cdot(7.25)
$$

In (7.24) and (7.25) there are the known relations [2] between "induction" $D^{1}$, field strength $E^{1}$ and field tensor component $F_{01}$

$$
D_{1}=\gamma_{11} D^{1}=\frac{2 \gamma}{\rho} \exp (\lambda / 2)=\frac{1}{\sqrt{g_{00}}} E_{1}, \quad E_{1}=F_{01}, \quad \gamma_{k l}=-g_{k l},(7.26)
$$

where $\gamma_{k l}$ is the space metric tensor with the determinant equal to $\gamma$. From (7.22) we find $F_{01}=-F_{10}$ components differed from zero

$$
F_{01}=-\frac{\partial A_{0}}{\partial \rho}=\frac{\gamma a_{0}}{\epsilon^{2}}\left(K_{1}(\alpha) I_{0}(\alpha)-I_{1}(\alpha) K_{0}(\alpha)\right) \cdot(7.27)
$$

Equating (7.27) to expression $F_{01}$ from (7.26) we find the coupling equation for functions $v(\rho)$ and $\lambda(\rho)$

$$
\exp \left(\frac{v+\lambda}{2}\right)=\alpha\left(K_{1}(\alpha) I_{0}(\alpha)-I_{1}(\alpha) K_{0}(\alpha)\right)=\frac{\rho}{2 \gamma} F_{01} \cdot(7.28)
$$

To find the second equation connecting these functions we shall consider the field force acting on the test charge $q$ fixed in the point with the coordinate $\rho$ from the filament axis. Let the mass of the test charge is $m_{0}$. Then one can to find the first curvature vector $F^{1}$ of the world line of this charge writting the comovement conditions for the fixed charges for metric (7.23) in the form

Whence we have

$$
\begin{gathered}
V^{k}=V_{k}=0, \quad V^{0}=\left(g_{00}\right)^{-1 / 2} \\
V_{0}=\left(g_{00}\right)^{1 / 2}, \quad F^{1}=F(\rho), \quad F^{0}=F^{2}=F^{3}=0 .(7.29)
\end{gathered}
$$

$$
F^{1}=\frac{1}{2} \frac{d v}{d \rho} \exp (-\lambda) \cdot(7.30)
$$

On the other hand, this quantity may also be found from the force acting on a charge due to the constraint holding the charge to be fixed in the field. This force is numerically equal to the force induced by the field and is opposite to it in sign.

$$
F^{1}=\frac{1}{2} \frac{d v}{d \rho} \exp (-\lambda)=-\frac{q}{m_{0} c^{2}} F^{10} V_{0}=-\frac{2 \gamma q}{m_{0} c^{2} \rho} \exp (-\lambda / 2),(7.31)
$$

From (7.27)-(7.31) we find

$$
\exp (v / 2)=-\frac{q}{m_{0} c^{2}} \int F_{01} d \rho=\frac{q A_{0}}{m_{0} c^{2}}+C_{1},(7.32)
$$

where $C_{1}$ is the constant of integration. We determine the constant of integration from the $A_{0}$ asymptotics at large distances from the filament. It is well known that in the classical representation $A_{0}$ potential at large distances from the filament is logarithmically diverged. For the considered situation it is not the case. Let us prove this. In the tetrads (5.9) on the basis of formulas (7.25), (7.26) $F_{(0)(1)}$ component of the electromagnetic field tensor has the form

$$
F_{(0)(1)}=\frac{2 Y}{\rho} \cdot(7.33)
$$

On the cylinder surface the field value $E_{0}$ coincides with the tetrad component $F_{(0)(1)}$. Therefore for the $\alpha$ value we have

$$
\alpha=\frac{\rho a_{n}}{2 c^{2}}=\frac{\rho \notin E_{n}}{4 m c^{2}}=\frac{\rho \notin \gamma}{R 2 m c^{2}}, \quad \rho \geq R \cdot(7.34)
$$

It follows from the analysis (7.34) that $\alpha \rightarrow \infty$ at $\rho \rightarrow \infty$. For the large distances from the filament one can to use the known asymptotic expansions for the Bessel functions [26]. 


$$
I_{0}(\alpha) ; \sqrt{\frac{1}{2 \pi \alpha}} e^{\alpha}, \quad K_{0}(\alpha) ; \sqrt{\frac{\pi}{2 \alpha}} e^{-\alpha} \cdot(7.35)
$$

This results in the expression for the component of 4-potential $A_{0}$

$$
A_{0}(\alpha)=\frac{\gamma}{\alpha}=\frac{2 m c^{2} R}{e \rho} \cdot(7.36)
$$

Hence this implyies that at $\rho \rightarrow \infty, A_{0} \rightarrow 0$. We proved that for our case the behaviour $A_{0}$ at infinity is sharply differed from the classical analog: instead of diverging value we obtained the vanishing value. This enables to determine the constant of integration $C_{1}$ from (7.32) from the demand of the Euclidean space at infinity. As a result we have

Using (7.28) we find

$$
\exp (v / 2)=1+\frac{q A_{0}}{m_{n} c^{2}} \cdot(7.37)
$$

$$
\exp (\lambda / 2)=-\frac{\rho}{2 \gamma} \frac{\partial A_{0}}{\partial \rho} \frac{1}{1+\frac{q A_{0}}{m_{0} c^{2}}} \cdot(7.38)
$$

For low fields at $\alpha=1$ using the known expansions for the modified Bessel functions [26]

we find

$$
I_{0}(\alpha) ; 1, \quad K_{0}(\alpha) ;-(\ln (\alpha / 2)+C),(7.39)
$$

$$
A_{0}(\alpha) ;-2 \gamma\left(\ln \left(\frac{\rho \pi_{0}}{2 \varepsilon^{2}}\right)+C\right), \quad-\frac{\partial A_{0}}{\partial \rho} ; \frac{2 \gamma}{\rho},(7.40)
$$

where $C=0.5772$. . is the Euler constant.

Expression (7.40) accurate within the value of the constants in the potential coincides with the classical statement. However the space-time metric even in the case of the low field remains as the Riemannian one, it is determined with the formula (7.37) and instead of (7.38) we have

$$
\exp (\lambda / 2)=\frac{1}{1+\frac{q_{0} A_{0}}{m_{0} c^{2}}} \cdot(7.41)
$$

Thus, for the three symmetry types (plane, spherical and cylindrical) the field energy created with the point charges does not diverge as in classical treatment and it is determined by the rest energy of the charges creating the field. Meanwhile the charge value is absent in the formulas for the energy.

Hereby, the proposed approach expands the field of application of the classical field theory when transition to the sufficiently small distances.

\section{Conclusions}

Enumerate the basic results obtained in the paper.

1. An exact static solution has been found for the charge field in a uniformly accelerated NFR realizable in Riemannian space-time. This solution allows one, in principle, to seek a space-time structure and to find fields from charged conductors of arbitrary shape. "Relativistic corrections" proved to be small for positively charged bodies, and the expressions for the scalar potential and the affine components of the field tensor coincide with the conventional ones in Minkowski space. It was found that for the bodies charged positively the "relativistic corrections" are small and the common electrostatics in the Minkowski space is true. These corrections may be considerable for negatively charged conductors or those being in an external electric field.

2. An exact expression has been found for the field and geometry of space-time outside a charged metallic ball. If the Coulomb repulsion force between charges on the ball surface is equal to the 
International Journal of Recent advances in Physics (IJRAP) Vol.3, No.2, May 2014

Newtonian attraction force and a similar equality is fulfilled between test particles outside the ball, then from our solution, to a high accuracy, follows Reissner-Nordström's well- known exact solution. When formal substitution of the coupling constants in the first approximation the Schwarzschild's solution is obtained.

3. An exact expression has been found for the energy of a point charge $W$, which is equal to $W=2 m c^{2}$ and independent of the quantum density of charge. This expression eliminates the main difficulty of classical and quantum electrodynamics leading to an infinite proper energy of a point charge. The expression for the energy shows that not all the mass of the charged elementary particle (electron or positron) has electromagnetic origin.

4. It is proved that the field energy ouside the infinite cylinder and filament is a finite quantity, which differs from the classical value of the energy that is infinitely large. In a close packing of charges at the cylinder surface (along the filament) we have an expression for the energy

$$
W=\frac{y h m c^{2}}{e}=N m c^{2}
$$

where $N$ is the number of electrons along the cylinder (filament) length $h$.

5. The possible effect of the electrostatic field presence inside the conducting charged cavity (the absence of the absolute screening) has been predicted. This work is an attempt to establish a link between the space-time curvature and the classical fields of bound structures. A lot of specific solutions connected with the radiation, electromagnetic wave propagation and the Doppler effect have been obtained in $[28,29]$.

\section{REFERENCES}

[1] Zommerfeld A.,(1958) “Electrodynamics”, Moscow.

[2] LandauL. D., LifshitsE. M., (1973) “Field theory,” Nauka, Moscow.

[3] Synge J. L.,(1960) “Relativity: the General Theory”, NHPC, Amsterdam.

[4] PodosenovS. A., (2000) "The space, time and the classic fields of the bound structures," Izd. "Sputnik+" Company, Moscow.

[5] PodosenovS. A., PotapovA. A., SokolovA. A., (2003) "The pulse electrodynamics of the wideband radio systems and the fields of the bound structures," "Radiotekhnika", Moscow.

[6] Potapov A.A.,(2002) "Fractals in radio physics and radiolocation", Logos, Moscow.

[7] Redzic D. V.,(2008) “Note on Devan-Beran-Bell's spaceship problem,”Eur. J. Phys.,Vol. 29, No.11.

[8] FoukzonJ., PodosenovS. A., PotapovA. A., "Relativistic length expansion in general accelerated system revisited," http://arxiv.org/pdf/0910.2298v1.

[9] PodosenovS. A., FoukzonJaykov, PotapovA. A., (2009) "Bell's Problem and a Study of Electronic Bunches in Linear Colliders,"Nelineiniy mir, Vol. 7, No. 8, pp. 612.

[10] PodosenovS. A., FoukzonJ., PotapovA. A., (2010) "A Study of the Motion of a Relativistic Continuous Medium," Gravitation and Cosmology, Vol. 16, No 4, pp. 307-312.

[11] Bell J. S., (1993) "Speakable and Unspeakable in Quantum Mechanics," Cambridge University Press, pp. 67.

[12] Gershtein S. S. and Logunov A. A., (1998)J. Bell's Problem. Particle and Nuclear Physics, 29, 5th issue.

[13] Podosenov S. A., (1982) in col. "Debatable problems of the theory of relativity and gravitation,"Nauka,Moscow, pp. 95.

[14] DeslogeE. A., (1989) Am. J. Phys., Vol. 57, No. 12, pp. 1121.

[15] DeslogeE. A., (1990) Int. J. Theor. Phys., Vol. 29, No. 2, pp. 193.

[16] RodichevV. I., (1974) “Gravitational theory in the orthogonal frame," Nauka, Moscow.

[17] Meller K.,(1975) "Theory of relativity," Transl. from engl., Atomizdat, Moscow.

[18] Fok V. A., (1961)“The theory of a space-time and the gravitation,” Fizmatgiz, Moscow. 
[19] Logunov A. A.,(1987) "Lectures on the theory of relativity and gravitation. The modern analysis of the problem," Nauka, Moscow.

[20] EinsteinA., (1965) "About the relativity principle and its consequences," Collection of the learned works, Vol. 1, Transl. from german, Nauka, Moscow, pp. 109.

[21] Podosenov S. A.,(1997) "Space-time structure and fields of bound charges,"Izvestyia Vuzov, Vol.40, No. 10, pp.63-74.

[22] Landau L. D., Lifshits E. M. (1982) “Electrodynamics of the continuous media,” Nauka, Moscow.

[23] IvanitskayaO. S., (1969) “Generalized Lorentz transformations and their application," Nauka i tekhnika, Minsk.

[24] VladimirovYu. S., (1982) “Reference frames in the gravitation theory,” Energoizdat, Moscow.

[25] Markov M.A., (1986) in col. “Theoretical-group methods in physics,” Vol. 1, Nauka, Moscow, pp.7.

[26] "Handbook on special functions,"(1979) Edited by Abramovich M. and Stigan I., Transl. from engl., Nauka, Moscow.

[27] Gradshtein I. S., Rizhik I. M., (1971) “Tables of integrals, sums, series and products," Nauka, Moscow.

[28] Potapov A. A., Podosenov S. A., Foukzon J. and Men'kova E. R., (2011) "New Method for Calculating Pulsed Electromagnetic Fields from Traveling Current Waves in Complex Wire Structures,"Physics of Wave Phenomena, Vol. 19, No. 2, pp. 1-12.

[29] Podosenov S. A., Foukzon J., and Potapov A. A., (2012)“Electrodynamics of a Continuous Medium in a System with Specified Structure,"Physics of Wave Phenomena, Vol. 20, No. 2, pp. 143-157.

\section{Authors}

Stanislav A. Podosenov (M'96) was born in Arkhangelsk, Russia in 1937. He received the degree in theoretical physics in 1963 and the Ph. D. degree in relativistic mechanics of deformable media in 1972, both from Moscow State University physical Faculty, Russia. From 1963 to 1970, he lectured physics at Moscow State Technical University, where he completed his postgraduate study. Since 1970, he has been working at the All-Russian Research Institute of Optophysical Measurements on problems of relativistic mechanics and electrodynamics, noninertial frames of reference in special and general relativity, and nonstationary neutron transfer theory. Presently, he is working in the field of EM pulses radiation theory and their interaction with conducting bodies. Dr. Podosenov is the laureate of prize of the Russian Federation government 2002 in the field of science and engineering.

Alexander A. Potapov was born in 1951. He is the Doctor of physical and mathematical Sciences. Dr. Potapov has been working as a chief researcher at the Institute of radio engineering and electronics of Russian Academy of Sciences named after V.A. Kotelnikov. He is working in the field of radio physics, dispersion and diffraction of electromagnetic waves, radiolocation, recognition and image and signal processing, determinate chaos, modern topology, fractal analysis, fractional operators, scaling effects.Dr. Potapov is the academician of Russian Academy of Natural Sciences RANS and the academician of Academy of Engineering Sciences named after A. M. Prokhorov. He is the professor of Kazan State Technical University named after A.N. Tupolev. Dr. Potapov is the Chief Editor of the magazine "Nonlinear world", Member of editorial board of the international magazine "Electromagnetic Phenomena". He is Honorable radio operator of the Russian Federation. Dr. Potapov is the author of the basic researches in the field of fractal approaches to radio physics, radio engineering and radar problems and broad spectrum of adjacent scientific and technology paths. He is working in this field from 1979. Dr. Potapov is the initiator of the pioneer researches and developments on fractal theory application in radio physics, scaling effects and fractal operators in radio systems in Russia. He is the author of more than 400 scientific publications, includes 10 monographs.

Jaykov Foukzon. Israel Institute of Technology, Haifa, Israel

Elena R. Men'kova was born in Moscow, Russia in 1955. She received the Ph.D. degree in 1984 from Russian Chemical-technological University, Russia.Since 1984 she has been working as a Junior Researcher, and then Senior Researcher at the All-Russian Research Institute of Optophysical Measurements. Her research interest focuses on the works connected with radiation and measurement of pulse electromagnetic fields. 